Journal of Engineering and Applied Sciences 14 (Special Issue 4): 7263-7269, 2019

ISSN: $1816-949 \mathrm{X}$

(C) Medwell Journals, 2019

\title{
Discrete Fracture Network and Fractured Reservoir Characterization in Khabaz Field-Tertiary Formation
}

\author{
${ }^{1}$ Adnan A. Abed and ${ }^{2}$ Sammera H. Mohammed \\ ${ }^{1}$ Department of Petroleum Engineering, University of Kirkuk, Kirkuk, Iraq \\ ${ }^{2}$ Department of Petroleum Engineering, University of Baghdad, Baghdad, Iraq
}

\begin{abstract}
Fracture reservoirs contain most of the oil reserves of the Middle East. Such reservoirs are poorly understood and recovery from fractured reservoirs is typically lower than those from conventional reservoirs loaded the fracture dip and dip azimuth data of fractures from 10 wells using Petrel 2013. Generated fracture intensity $\log$ and interpreted fracture data was used to make stereonet plots to study the fracture orientation. The N130 fracture repartition in the tertiary is intimately related to the proximity of large faults and the maximum density is reached in a crestal position. Fracture density increases over some structural surfaces as Jeribe and Anah limestones which systematically appear as the most competent/"fracturable" formations. Permeability expected consistency between the fracture corridor occurrence and the highest values of permeability. The permeability ranges between 2 and $1000 \mathrm{mD}$. Results of fracture upscaling; the fracture porosity ranges between 0.0004 and 0.06 p.u. fracture-matrix coupling (sigma) ranges between 0 and $11 / \mathrm{ft}^{2}$.
\end{abstract}

Key words: Fractured modeling, DFN, intensity, Middle East, fracture, appcar, sustematically

\section{INTRODUCTION}

Fractured reservoirs are challenging to model due to the complexity of processes involved in the construction and preservation of the fracture network as well as the heterogeneity reservoir. An understanding of stress directions and natural fracture patterns within the basin are imperative in well design and completion. By exploiting natural fractures with an optimum well design, an operator can ensure maximum hydrocarbon recovery. The fracture model is used to build a dual porosity model in Petrel that incorporates both matrix and fracture properties. Using regional fracture trends and available field data a fracture model for tertiary formation in Khabaz field.

In this study, the discrete fracture network modeling provides a geologically sound representation based on parameters (fracture orientation, length, aperture) and the means for up scaling is to a dual medium model that can be simulated using it is a stochastic representation that can be used to estimate the uncertainty and the heterogeneity of the output variables (fracture porosity, permeability, etc.)

Discrete fracture networks will be upscale into the developed matrix model of similar resolution by Oda (1985) to generate the fracture porosity, the three permeability tensors and the matrix to fracture coupling factor.
Theoretical background

Discrete fracture network models: Dershowitz et al. (2000), Wei (2000), Makel (2007) and Spence et al. (2014). The Discrete Fracture Network (DFN) approach is commonly used to estimate fracture permeabilities. DFNs are stochastic representations of the fracture network constrained by a wide range of reservoir data. The DFN approach is an efficient and geologically consistent way, to model multiscale fractures as it can capture the connectivity and scale dependent heterogeneity of the fracture system (Dershowitz et al., 2000; Wei, 2000; Makel, 2007; Spence et al., 2014). In a DFN Model, fractures are represented by planar elements. To build a DFN constrained by deterministic observations of fractures, five geometrical fracture characteristics that control the interconnectedness of the subsurface fracture network are required. These characteristics include the fracture intensity/density, orientation, aperture, length and aspect ratio. Multiple equiprobable realisations of the fracture system can then be generated stochastically with a given set of input parameters to account for uncertainties in the fracture network characterisation. For example, the fracture intensity in a given reservoir may be generated as a result of faulting and hence cluster around faults (fault related intensity), may be part of a more stratigraphically confined fracture system giving rise to layered high fracture permeability (bedding related

Corresponding Author: Adnan A. Abed, Department of Petroleum Engineering, University of Kirkuk, Kirkuk, Iraq 
intensity) or part of a pervasive background fracture system (regional intensity). Analysing the influence of fracture intensity scenarios on hydrocarbon production in a given reservoir will enable us to link flow patterns to the fracture network characterisation, especially, for EOR simulation. Hence, a systematic and integrated analysis of the fracture network geometry can provide a pathway to better understand the fracture system, fracture contribution to subsurface fluid displacement, the best way to accurately model the fracture flow impact and to develop the reservoir accordingly.

Discrete fracture network upscaling: In standard reservoir simulation applications that use conventional finite difference formulation, the DFN fracture representation is employed in dual continuum models. The effective properties for the fracture medium are obtained via. analytical or flow based fracture upscaling methods (Dershowitz et al., 2000). Analytical upscaling methods such as the Oda (1985) method are usually preferred for field-scale applications due to the significant computational efficiency compared to flow-based upscaling but can be inaccurate for poorly connected fracture networks (Elfeel and Geiger, 2012). Generally, the upscaling of the DFN must be done with utmost care to ensure that geological uncertainties captured in the DFN are not masked by uncertainties in the upscaling step. The effective fracture porosity, $\varphi f$ is calculated as Eq. 1:

$$
\varphi f=P 32 \times a f
$$

where, P32 represents the volumetric fracture intensity (total fracture area per unit volume) and a f denotes the fracture aperture.

Oda fracture permeability upscaling: Oda (1985) to compute the effective fracture permeability for a specific simulation grid cell from a DFN Model, a permeability tensor, $F_{i j}$ which denotes flow along the fracture's unit normal, $\mathrm{n}$, needs to be estimated. The tensor is calculated by summing over the individual fractures, $\mathrm{f}$ in the grid cell provided the fracture area, $\mathrm{A}_{\mathrm{f}}$ and transmissibility, $\mathrm{T}_{\mathrm{f}}$ are known Eq. 2:

$$
\mathrm{F}_{\mathrm{ij}}=\frac{1}{\mathrm{~V}} \sum_{\mathrm{f}=1}^{\mathrm{N}} \mathrm{A}_{\mathrm{f}} \mathrm{T}_{\mathrm{f}} \mathrm{n}_{\mathrm{if}} \mathrm{n}_{\mathrm{jf}}
$$

where, the number of fractures is denoted by $\mathrm{N}$, the fracture unit normal representing its direction and orientation is represented by $\mathrm{n}$ and the total fracture pore volume is represented by $\mathrm{V}$. If $\mathrm{F}_{\mathrm{ij}}$ is rotated into the planes of the permeability tensor by multiplication with $\delta_{i j}$, the fracture permeability can be approximated as (Dershowitz et al., 2000) Eq. 3:

$$
\mathrm{k}_{\mathrm{ij}}=\frac{1}{12}\left(\mathrm{~F}_{\mathrm{ff}} \delta_{\mathrm{ij}}-\mathrm{F}_{\mathrm{ij}}\right)
$$

where, $F_{\mathrm{ff}}$ defines the principal directions of the permeability. The application of Oda's method is based on equation above is the assumption that fractures of any length will contribute to the upscaled permeability even if fractures do not form a percolating network in the grid block. Therefore, a modified version of Oda's method was introduced for situations where fractures are not connected in a grid block (Anonymous, 2010) Eq. 4:

$$
k_{i j}=M\left(F_{f f} \delta_{i j}-F_{i j}\right)-M C\left(F_{f f} \delta_{i j}-F_{i j}\right), C \geq C_{0}
$$

Where:

$\mathrm{M}=\mathrm{A}$ multiplier for scenarios where fractures are not connected

$\mathrm{C}_{\mathrm{O}}=$ Determines the threshold fracture connectivity

\section{MATERIALS AND METHODS}

Loading data and fracture analysis: Loading and fracture data analysis is the next step in the reservoir characterization and modeling workflow. Loaded the fracture dip and dip azimuth data of fractures from 10 wells in the Petrel project depend on previous study of that based upon Qara Chauq anticline analogy to build a structural framework (faults and fractures network). Generated fracture intensity $\log$ in these wells and analysis of fracture by tadpole concentration at wells interpreted fracture data was used to make stereonet plots for tertiary formation in Khabaz field to study the fracture orientation. The general directional rose diagram indicates that the major part of the fractures is parallel to the fold axis. Statistically, this direction tends to appear over-represented with respect to the other trends (Fig. 1 and 2).

Fracture network modeling: After loading and fracture analysis data the discrete fracture network modeling representation provides a geologically sound representation based on physically measurable parameters (fracture orientation, length, aperture) and the means for up scaling is to a dual medium model that can 
J. Eng. Applied Sci., 14 (Special Issue 4): 7263-7269, 2019

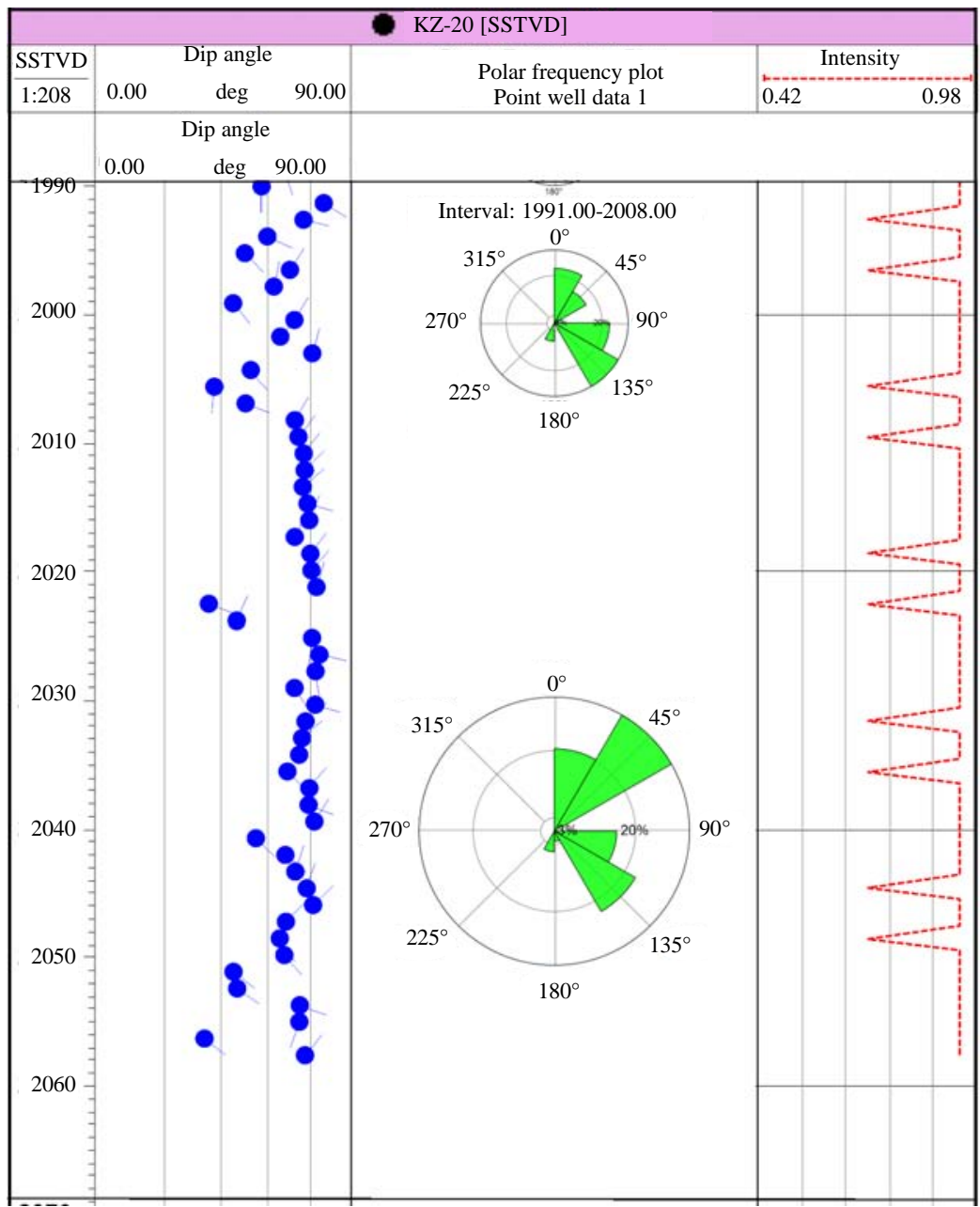

Fig. 1: Tadpole and rose diagram for dip and azimuth of fracture in KZ-20

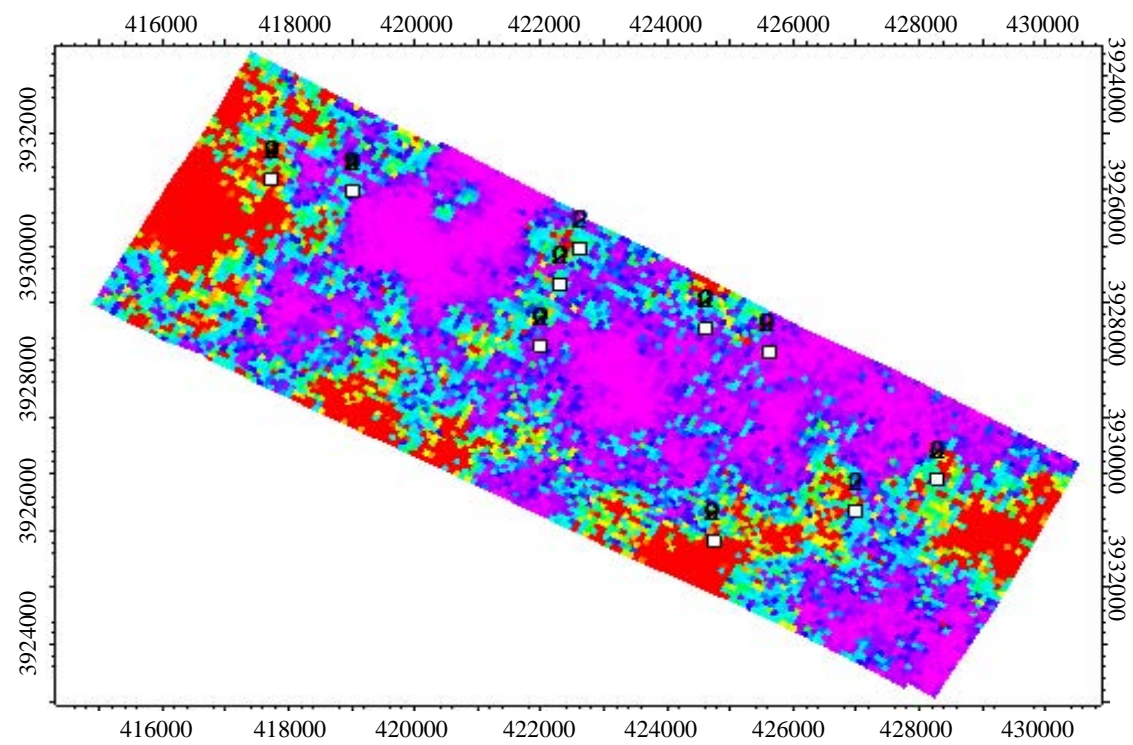

Fig. 2: Continue 


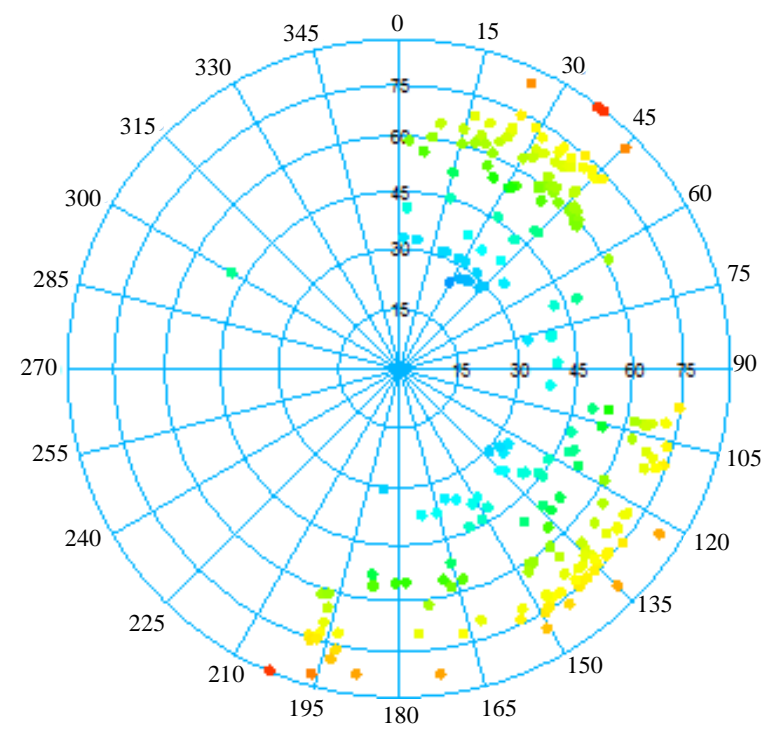

Fig. 2: a) 2D intensity map and stereonet plot of all fracture dip angle and b) dip azimuth in tertiary reservoir

be simulated using it is a stochastic representation that can be used to estimate the uncertainty and the heterogeneity of the output variables (fracture porosity, permeability, etc.). Discrete fracture network modeling was constructed depend on fracture distribution, geometry, orientation and fracture aperture.

\section{RESULTS AND DISCUSSION}

Fracture distribution: Fracture density measurement is crucial in the discrete fracture network model generation, to be able to quantify the fracture intensity in a $3 \mathrm{D}$ grid.

Fracture orientation: Finally, the orientation of the fractures in terms of dip angle and azimuth is defined according to the well data; the choice of the distribution is done on the stereonet of the sets of potential flow contributing fractures with specific orientation. A different orientation is defined for each fracture set.

Fracture aperture: Fracture aperture is a perpendicular width of the fracture. Previous research showed that the fracture aperture distribution follows lognormal distribution (Keller, 1996; Gale, 1987). The lognormal distribution is described by the mean and standard deviation.
Applying the approach for discrete fracture network, field data was analyzed. Overall, the trend of all natural fracture orientations is NE-SE and some SE-SW, (Fig. 3a, b).

Three different fracture scales have been referenced generally traducing 4 principal direction trends; N135 Normal faults (identified throw values from 10-100 m) in segmented arrays, N45 and N135, fracture swarms and background diffuse fractures (N15, N45, N105, N135). The N130 fracture repartition in the tertiary is intimately related to the proximity of large faults and the maximum density is reached in a crestal position. This direction constitutes the best represented trend over the study area.

The N35 fracture system should have a systematic distribution (tensile jointing) under the early beginning of the NE-SW shortening. Diffuse fractures and significant fracture swarms theorically affect tertiary by the same deformation processes. Fracture density increases over some structural surfaces as Jeribe and Anah limestones which systematically appear as the most competent "fracturable" formations. The visible part of the Azkand (reefal facies) emerges less fractured (Fig. 4).

Discrete fracture network upscaling: A discrete fracture network model is made with some fracture network attributes as described above. However, for 
(a)
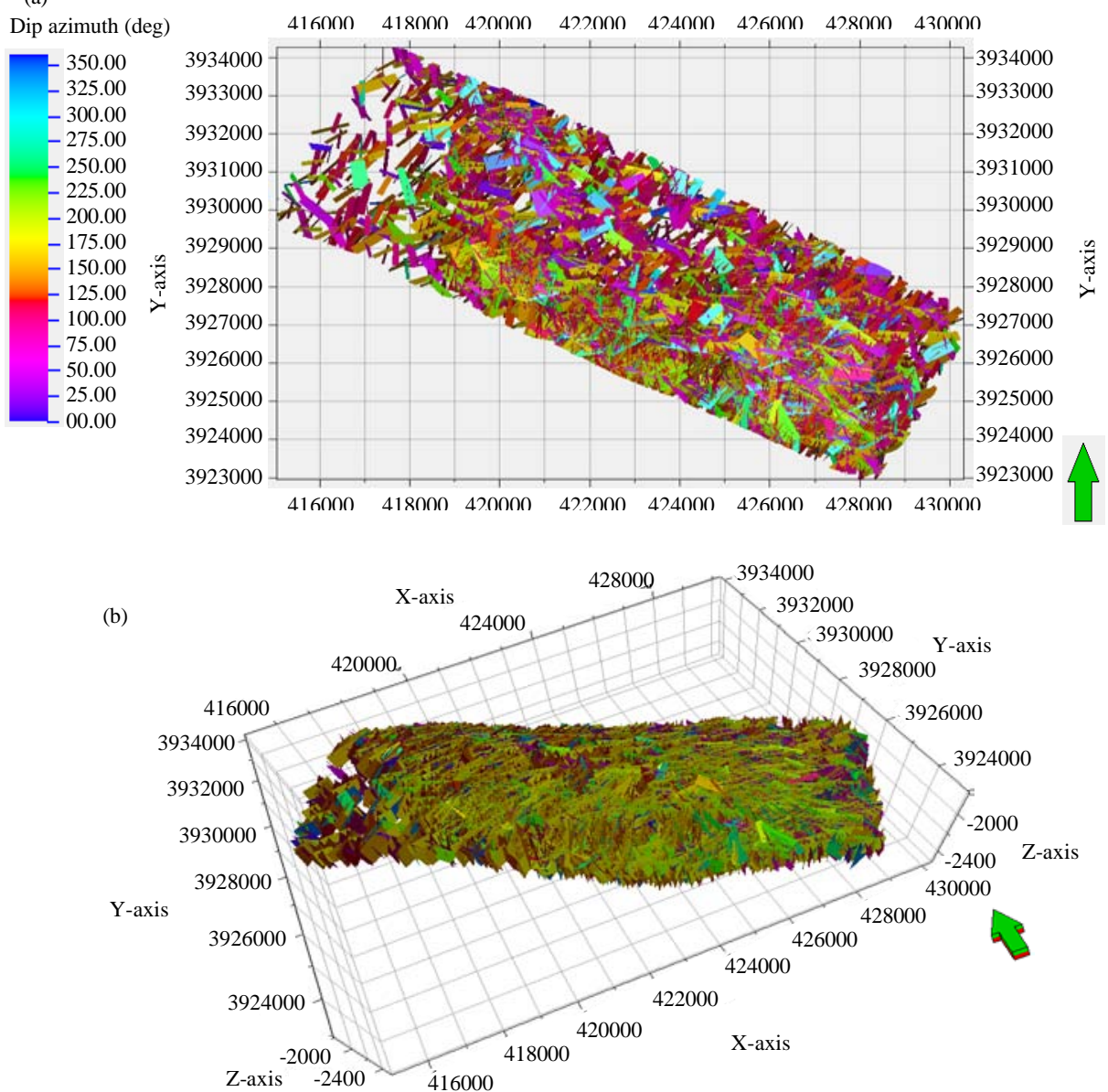

Fig. 3: a) 2D dip azimuth of fracture in tertiary reservoir and b) 3D dip azimuth of fracture in tertiary reservoir
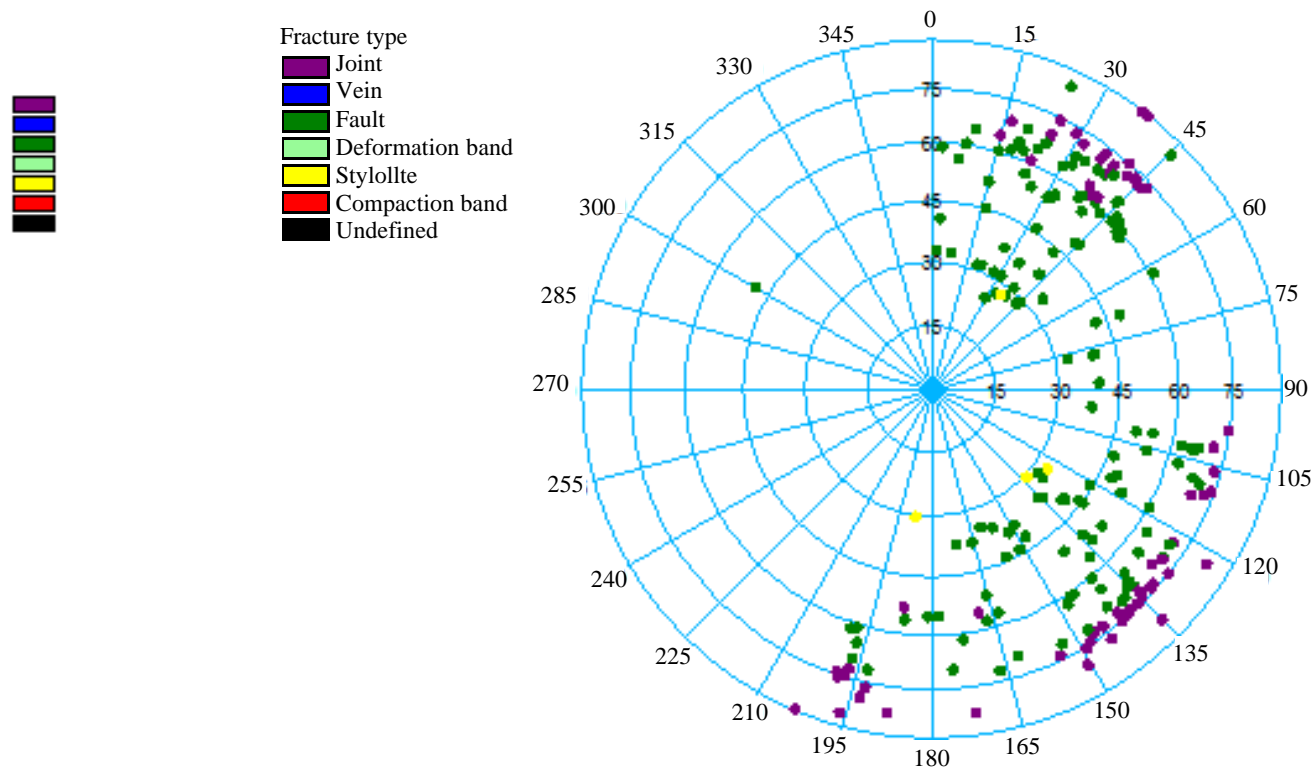

Fig. 4: Streonet plot of fracture type in tertiary reservoir 


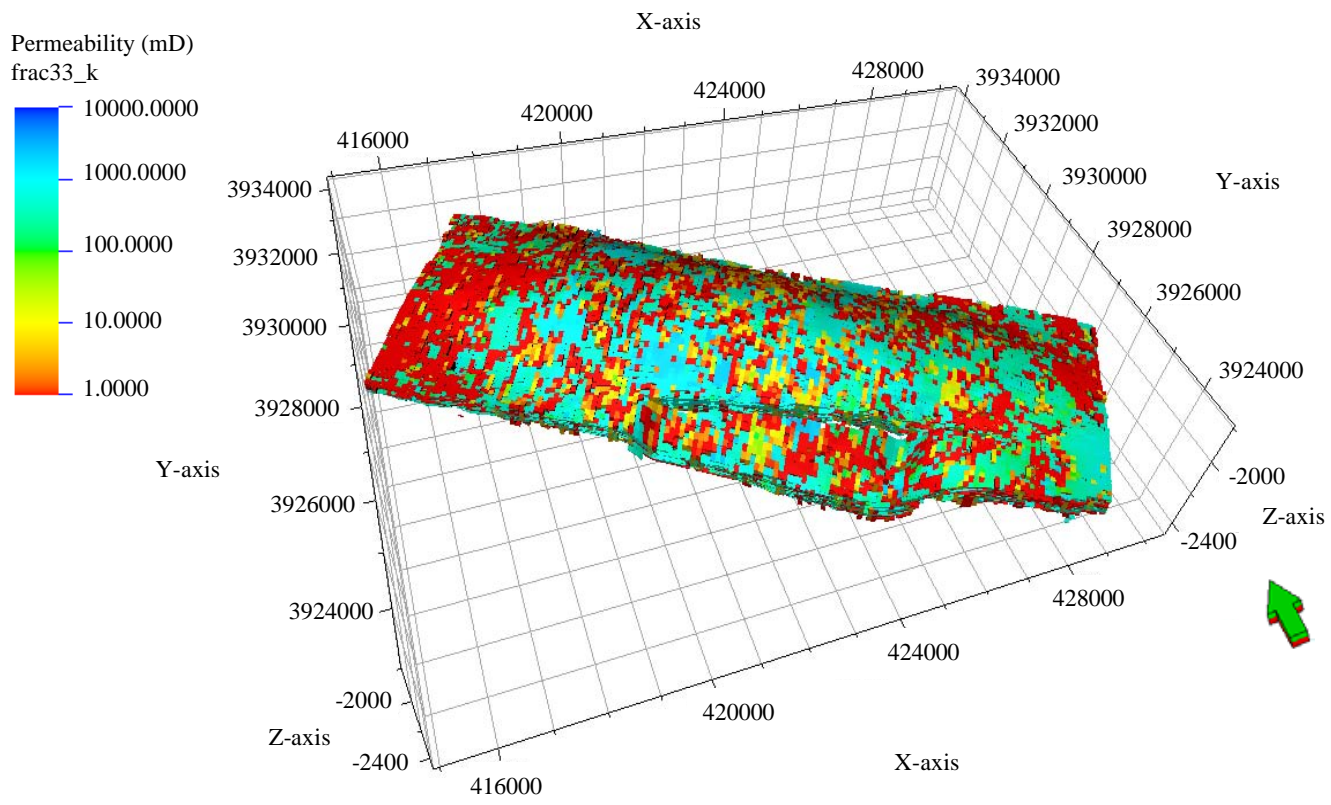

Fig. 5: 3D fracture permeability in tertiary reservoir

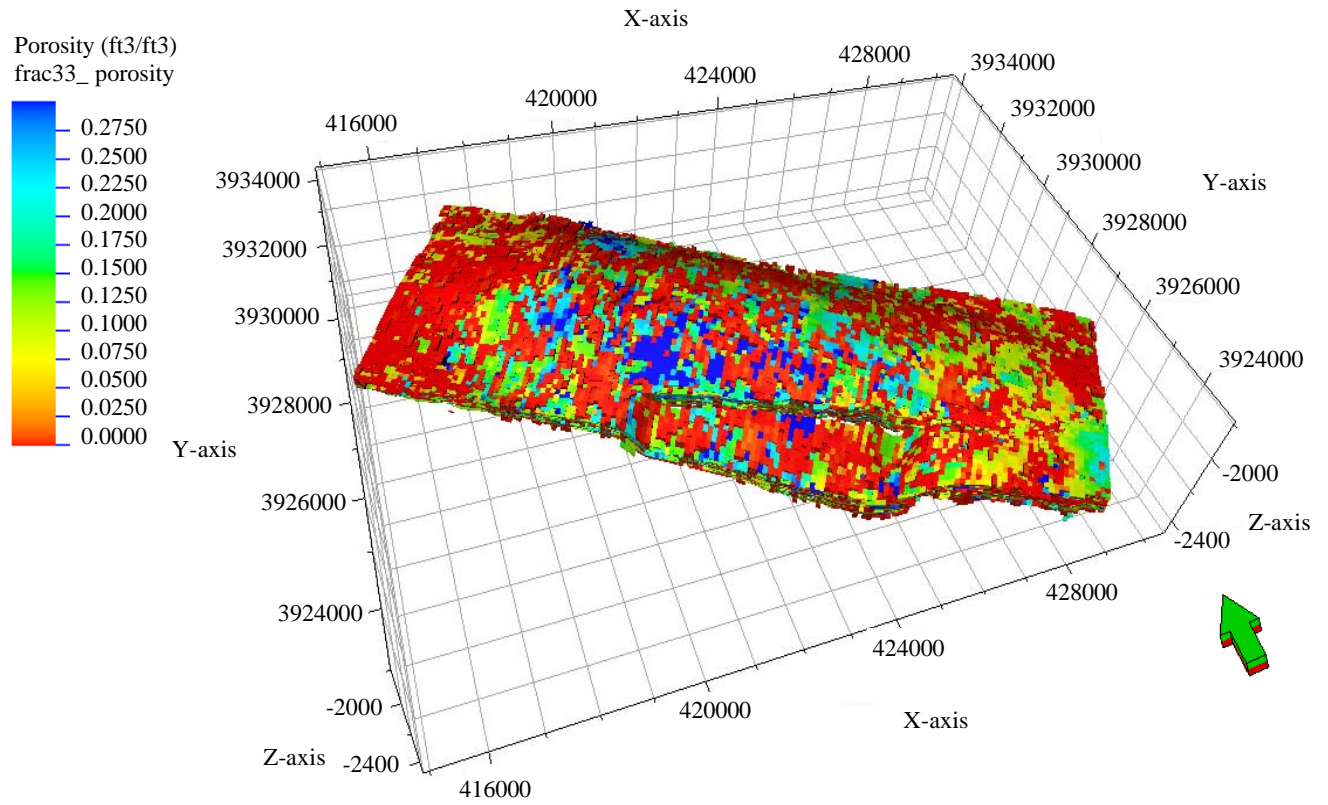

Fig. 6: 3D fracture porosity in tertiary reservoir

practical purposes in dynamic reservoir models, these are not useful, until they are upscaled into the required grid properties that can be used for simulation. Each of these discrete fracture networks were upscaled into the already developed matrix model of similar resolution by Oda (1985) to generate the fracture porosity, the three permeability tensors and the matrix to fracture coupling factor.
Property modeling incorporating fracture network: Results of fracture upscaling: permeability expected consistency between the fracture corridor occurrence and the highest values of permeability. The permeability ranges between 2 and $1000 \mathrm{mD}$. Results of fracture upscaling; the fracture porosity ranges between 0.0004 and 0.06 p.u. fracture-matrix coupling (sigma) ranges between 0 and $11 / \mathrm{ft}^{2}$ (Fig. 5-7). 


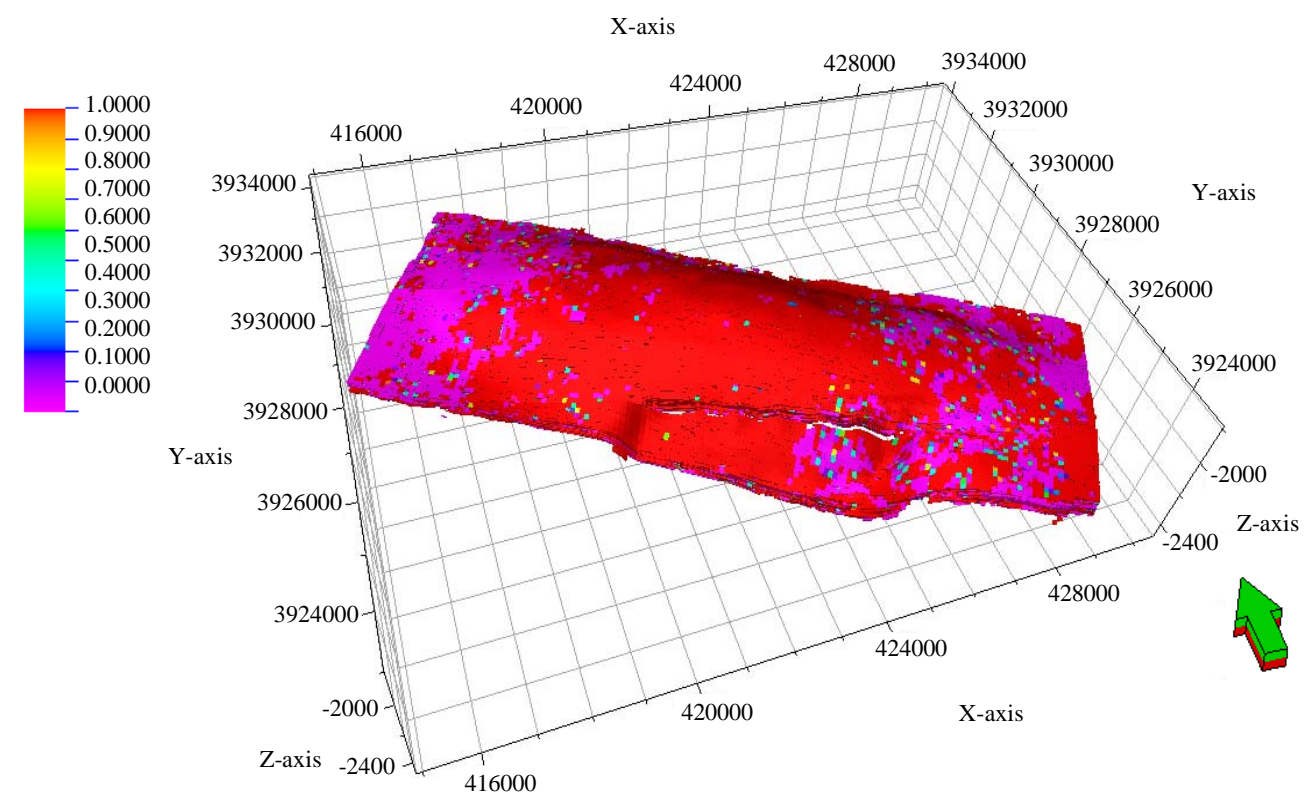

Fig. 7: 3D matrix- fracture coupling (sigma) in tertiary reservoir

\section{CONCLUSION}

Discrete fracture network modeling and fracture characterization were constructed depend on fracture distribution, geometry, orientation and fracture aperture which means for up scaling is to a dual medium model that can be used to estimate the uncertainty and the heterogeneity of the output variables (fracture porosity, permeability, etc.).

The N130 fracture repartition in the tertiary is intimately related to the proximity of large faults and the maximum density is reached in a crestal position. This direction constitutes the best represented trend over the study area.

Fracture density increases over some structural surfaces as Jeribe and Anah limestones which systematically appear as the most competent/ "fracturable" formations. The visible part of the Azkand (reefal facies) emerges less fractured.

\section{REFERENCES}

Anonymous, 2010. FracMan version 7.4 user manual. Golder Associates Incorporated, Redmond, Washington.

Dershowitz, B., P. Lapointe, T. Eiben and L. Wei, 2000. Integration of discrete feature network methods with conventional simulator approaches. SPE. Reservoir Eval. Eng., 3: 165-170.
Elfeel, M.A. and S. Geiger, 2012. Static and dynamic assessment of DFN permeability upscaling. Proceedings of the SPE Europec/EAGE Annual Conference, June 4-7, 2012, Society of Petroleum Engineers, Copenhagen, Denmark, pp: 1-16.

Gale, J.E., 1987. Comparison of coupled fracture deformation and fluid flow models with direct measurements of fracture pore structure and stress-flow properties. Proceedings of the 28th U.S. Symposium on Rock Mechanics (USRMS), June 29-July 1, 1987, American Rock Mechanics Association, Tucson, Arizona, pp: 1-10.

Keller, A.A., 1996. Single and multiphase flow and transport in fractured porous media. Ph.D Thesis, Stanford University, Stanford, California.

Makel, G.H., 2007. The modelling of fractured reservoirs: Constraints and potential for fracture network geometry and hydraulics analysis. Geol. Soc. London Spec. Publ., 292: 375-403.

Oda, M., 1985. Permeability tensor for discontinuous rock masses. Geotech., 35: 483-495.

Spence, G.H., G.D. Couples, T.G. Bevan, R. Aguilera and J.W. Cosgrove et al., 2014. Advances in the study of naturally fractured hydrocarbon reservoirs: A broad integrated interdisciplinary applied topic. Geol. Soc. London Spec. Publ., 374: 1-22.

Wei, L., 2000. Well test pressure derivatives and the nature of fracture networks. Proceedings of the SPE International Conference and Exhibition on Petroleum, February 1-3, 2000, Society of Petroleum Engineers, Villahermosa, Mexico, pp: 1-7. 